\title{
Verification of Medium Range Weather Forecast for Udham Singh Nagar Region in order to Improve Methodology Followed
}

\author{
Shivani Kothiyal ${ }^{1}$, R.K. Singh ${ }^{1}$, A.S. Nain ${ }^{1}$, Himani Padalia ${ }^{2}$ and Gaurav Chaturvedi ${ }^{1}$ \\ ${ }^{1}$ Department of Agrometeorology, Govind Ballabh Pant University of Agriculture and \\ Technology, Pantnagar 263145, Uttarakhand, India \\ ${ }^{2}$ District Consultant, Rajasthan Grameen Ajeevika Vikas Parishad, Ministry of Rural \\ Development, Kota 324001, Rajasthan, India \\ *Corresponding author
}

\section{A B S T R A C T}

\section{Keywords}

Medium range weather forecast,

Forecast verification, Skill scores, Statistical interpretation.

Article Info

Accepted:

15 October 2017

Available Online:

10 December 2017
Medium range weather forecast was provided by IMD using T-80 model at district level for five days in advance. The forecast was provided for seven weather parameters and accurate forecasting of these continues to be a major challenge for scientific community. The forecast was provided biweekly, Tuesday and Friday while the observed data for the same is collected from IMD approved agrometeorological observatory situated at different SAUs. IMD criteria was followed for assignment of correct, usable and unusable percent and different scores along with statistical interpretation are calculated to test the weather forecast for the weather parameters during 2016-17. The rainfall represented an average good forecast over the year. The overall cloud forecast for all the seasons was good with high correct percent for all the seasons. The wind speed and direction depicted poor forecast for all the seasons over the year. Maximum temperature had a high usable percent over the year. The overall forecast for minimum temperature was slightly good. FAR was the correct skill score for False alarms while FAR in combination with POD represented a reliable skill score for rainfall forecast. The chi square values too provided with reliable results for the temperature forecast.

\section{Introduction}

The malevolent effect of weather may be partially reduced if the occurrence of the events is predicted well in advance and farmers are suitably advised to take ameliorative measures to reduce their effects. For this purpose, weather forecasting is an effective task taken up by India Meteorological Department (IMD) which provides weather data along with 5 days advance weather forecast to the farmers through Agromet Advisory Service under Gramin Krishi Mausam Sewa (GKMS). These Services are required to prepare an integrated Agromet Advisory bulletin based on the 5 day medium range weather forecast received from IMD with the help of experts from different disciplines viz. Agronomy, Horticulture, Plant Pathology and Entomology, Vegetable Science and Veterinary and Animal Sciences. These bulletins are then provided to the farmers twice a week; accordingly they can take prior actions to prevent losses up to some extent. Weather forecasting is a prediction of what the weather will be like in an hour, tomorrow, week, next week or season. It involves a combination of computer models, observations and knowledge of trends and 
patterns of weather. By using these models, reasonable accurate forecasts can be made for now casting (few hrs to one day), short range (1-3 days), medium (3-10 days) and long range periods (month/season). An estimate made by the agribusiness, a community in western countries, indicates that the forecast can be put to economical use if it is 50 to 60 percent correct (Seeley, 1994). An agriculture relevant forecast is not only useful for efficient management of farm inputs but also leads to precise impact assessment (Gadgil, 1989), also an aberrant or unfavourable weather events such as drought, flood, cold wave and heat wave, etc. cause a great reduction in production. Hence, the verification process provides with the information regarding accuracy of weather forecast and how it can be effectively improved in order to help the farmers. Damrath et al., (2001) reported that the statistical interpretation methods are used to increase the reliability of the precipitation forecast. Accurate forecasting of rainfall patterns and other weather variables continue to be a major challenge for scientific community. The emerging capacity to provide timely, skillful weather forecasts offers the potential to reduce vulnerability to vagaries of weather (Hansen, 2002). Accuracy of weather forecast for western agro climatic zone of Haryana during kharif season was reported by Khichar and Bishnoi (2003). They have reported that more than 60 percent farmers realized the weather prediction and agrometadvisories to be useful for irrigation scheduling, pest/diseases management and harvesting of crops.

\section{Materials and Methods}

The study was conducted at the agrometeorological observatory situated in Norman E. Borlaug Crop Research Centre in Govind Ballabh Pant University of Agriculture and Technology from where the daily observed weather data for the Udham Singh Nagar district was collected for the year 2016-17. The forecasted weather data is provided by India Meteorological Department (IMD), New Delhi on its website www.imdagrimet.gov.in or by Regional Meteorological Centre (RMC), Dehradun who value adds the data provided. The value addition to the T-80 model provided forecast is done manually by considering the climatology of the region, products of other NWP models, prevailing synoptic condition of the region and neighbourhood, satellite imageries on the day of forecast, DWR products on the day of forecast and knowledge gathered by comparing model forecast with the observed data in the previous years. The location specific medium range weather forecast is received regularly from IMD, New Delhi on every Tuesday and Friday.

This value added weather forecast data was verified against the observed data using skill scores and statistical parameters. In accordance to the farmers the important six parameters that are rainfall, wind speed and direction, maximum and minimum temperature and cloud cover were verified. Maximum skill scores for the rainfall forecasts were used for the determination of forecast accuracy while and additional statistical parameter chi-square was utilized for temperature. Ratio score and hanssenkuipers score have been used till now effectively but some new scores have been introduced in order to detect the accuracy of the forecast. The following dissemination procedure can be understood from the flowchart in Figure 1. The skill scores used in the verification include Ratio score that is also known as Forecast Accuracy (ACC) or Hit Score or Percentage Correct and it measures the proportion of correct forecasts. It varies from 0 to 100 where 100 represent the perfect forecasts. The HK score gives equal emphasis 
to the ability of the forecast model to correctly predict events and non-events. The score has a range of -1 to +1 with 0 representing no skill. Probability of Detection (POD) is the ratio of correct rain forecast. It uses only the observed events. POD is sensitive only to missed events and not False alarms. POD score ranges from 0 to 1 with a perfect score at 1 and no skill at 0 . False alarm rate (FAR) is not sensitive to missed events. Its range is 0 to 1 with a perfect score at 0 . The Heidke Skill Score ranges from -1 to +1 . It measures the fraction of correct forecasts after eliminating those forecasts which would be correct due to purely a random chance. The RMSE value for all six major weather parameters is worked out for the absolute error between observed and forecasted weather data while correlation coefficient helps in determining the skill of the forecast by evaluation of the relationship between the predicted and observed values. Chi square values for each day forecast for the maximum and minimum temperature are worked out to explain the significant or nonsignificant relationship between the daily predicted and observed data values for all the four seasons. These scores are calculated in reference to table 1 .

In Table 1:

$\mathrm{Y}=\mathrm{Yes}$ and $\mathrm{N}=\mathrm{No}$

First letter in the pair is observed rainfall while the second depicts the predicted rainfall.

YY $(\mathrm{H})=$ No. of hits (Rainfall has been observed as well as forecasted)

NY $(F)=$ No. of false alarms (Rainfall has been predicted but not observed)

YN $(\mathrm{M})=$ No. of misses (Rainfall has been observed but not predicted)
NN $(Z)=$ No. of correct predictions of no rain (neither predicted nor observed)

Total no. of cases is given by $\mathrm{N}$ and this also represents the number of days for which the forecast is given.

$\mathrm{RS}=(\mathrm{YY}+\mathrm{NN}) /(\mathrm{YY}+\mathrm{NN}+\mathrm{YN}+\mathrm{NY})$

$\mathrm{HK}=(\mathrm{NN} * \mathrm{YY}-\mathrm{NY} * \mathrm{YN}) \quad /$ $(\mathrm{NN}+\mathrm{NY}) *(\mathrm{YN}+\mathrm{YY})$

$\mathrm{POD}=\mathrm{YY} / \mathrm{YY}+\mathrm{YN}$

$\mathrm{FAR}=\mathrm{NY} / \mathrm{YY}+\mathrm{NY}$

$\mathrm{HSS}=((\mathrm{NN} * \mathrm{YY})-(\mathrm{NY} * \mathrm{YN})) /[((\mathrm{NN}+$ $\mathrm{YN}) *(\mathrm{YN}+\mathrm{YY}))+((\mathrm{NN}+\mathrm{NY}) *(\mathrm{NY}+$ $\mathrm{YY}))] / 2$

$\mathrm{R}\left(\mathrm{f}, \mathrm{o}_{\mathrm{i}}\right)=\frac{\Sigma\left(f_{\mathrm{i}}-\bar{f}\right)\left(o_{\mathrm{i}}-\bar{o}\right)}{\left[\Sigma\left(f_{\mathrm{i}}-\bar{f}\right)^{2} \Sigma\left(o_{\mathrm{i}}-\bar{o}\right)^{2}\right]^{1 / 2}}$

$\mathrm{RMSE}=\sqrt{\frac{1}{n} \sum\left(f_{i}-o_{i}\right)^{2}}$

$\chi^{2}=\left\{(\mathrm{Oi}-\mathrm{Pi})^{2}\right\} / \mathrm{Pi}$

Where,

$\mathrm{f}_{\mathrm{i}}=$ forecast value

$\bar{f}=$ mean forecast value

$\mathrm{o}_{\mathrm{i}}=$ observed value

$\bar{o}=$ mean observed value

$\mathrm{n}=$ total number of observations

$\chi^{2}=$ Chi-square

Oi $=$ Observed values for each day of each month in a season

$\mathrm{Pi}=$ Predicted values for each day of each month in a season 


\section{Results and Discussion}

The comparative forecast for the entire year 2016-17

\section{Rainfall}

Table 2 shows the comparative and average values of different parameters during the year. The maximum correct percentage for the forecast has been obtained in winter, 96 followed by summer, 95, post monsoon, 93 and monsoon, 45. This is due to the maximum rainfall that occurred in the monsoon months from June- September in which the south-west monsoon dominates along with variation in quantity between predicted and observed values is very high while the other seasons having few forecasts of rainfall. Though, the rainfall prediction should be more accurate during the monsoon season when compared to other seasons since kharif season is most important for crops from farmer point of view. The usable percent as obtained for the monsoon season, 23 while 7,2 and 3 percent for the post monsoon, winter and summer season respectively. The unusable percent was highest for the monsoon season, 32 along with 0,2 and 2 for post monsoon, winter and summer season respectively. The unusable percent was highest during the monsoon season, thus it needs to be improved. This depicts the forecast is found to be very good for all the seasons with the highest average percent for the correct forecasts, 78 followed by unusable percent as 12 and usable percent as 10 percent.

The variation of skill scores is presented in Figure 2. The average for HSS score and HK score is 0.135 and 0.247 respectively which clearly represents a very little skill in the forecast. The RS average is 75 percent representing the score very near to 100 percent in most of the seasons. The average is 0.850 and 0.537 respectively for FAR and POD representing the false alarms to be more than the perfect forecasts.

The mean value for correlation, 0.197 represents positive but a weak correlation between forecasted and observed values. The overall results obtained represent a good forecast for the winter season with the poor forecast in the post monsoon season. There is a need for improvement in the forecasts for the post monsoon season. The RMSE average, $8.584 \mathrm{~mm}$ represents the moderate deviation and variation in the data values taken. The predicted values are higher than the observed for the monsoon, post monsoon and summer season. The total forecast for the year results to be a good one and needs some improvement. Almost similar results were obtained by Mummigatti et al., (2013). The skill scores worked efficiently for the monsoon season where maximum rainfall was predicted as well as observed. The HSS, FAR and POD together meant to be good scores for depiction of usable and unusable rainfall forecasts during the year.

\section{Cloud cover}

Table 3 clearly presents the comparative and average values of different parameters during the year 2016-17. The correct percentage is highest for the post monsoon season while for the other season it is nearly same for all, thus depicting the forecasts to be very good if usable forecasts are also taken into account. The average percent gives the highest value for the correct forecast as 52 followed by unusable and usable average as 24 . The average correlation value, 0.353 depicts a good positive relation between forecasted and observed values. Average RMSE, 2.736 Octa represents the low error and low deviation in data. Predicted values are higher than the observed one for the monsoon, winter and 
summer season. The forecast is good for the whole year with the best forecast for the post monsoon season.

\section{Wind speed}

Comparative and average values of different parameters during the year can be observed in Table 4. Here, the highest average percent is for the unusable which is 56 followed by the usable percent as 31 percent and correct percent, 13. This depicts the poor forecast for wind speed with a low average correlation which is positive and very weak with the value of 0.134 . The swing of correlation is towards negative for the post monsoon season depicting again a poor forecast. The average RMSE value of $4.849 \mathrm{~km} / \mathrm{h}$ depicts the moderately high error with high difference between the forecasted and observed values for the whole year. The predicted values are higher than the observed for the monsoon, post monsoon and winter season. The overall forecast for the year appears to be moderately poor. The variations in statistical parameters for wind speed have been depicted in Figure 2.

\section{Wind direction}

Table 5 depicts the comparative and average values of different parameters during the year 2016-17 for wind direction. The forecast represents the high unusable percent as $48 \%$ followed by the average percent for usable, 11 with a small difference for the correct percent as 41. This together gives a slightly good forecast for the year if correct and usable are used together. Accordingly the average correlation as 0.304 represents a positive but a slightly weak relation between forecasted and observed values. Average RMSE is $123^{\circ}$ which represents a high error and large difference between the observed and predicted values with large deviation in the data. Post monsoon and winter season show the predicted values to be higher than the observed. The overall forecast for the year resembles to be poor for the wind direction.

\section{Maximum temperature}

Comparative and average value for the parameter during the year 2016-17 is presented in Table 6 . The average for the correct percentage of the forecast is highest, 45 followed by unusable as 33 percent and thereafter the usability percent as 22. This depicts the correct forecast, thus determining the forecast to be good for the year. While for the post monsoon and monsoon season forecasts seems to be good due to their high correct percent. The correlation coefficient with the value of 0.724 represents the positive and high correlation between the observed and predicted values. The average RMSE value 2.371 represents a low error along with a low difference between the values. The correlation coefficient value during the summer season for the maximum temperature is positive with the value as 0.860. This value is highest giving an extremely strong relationship between the forecasted and observed data and at the same time with very high unusable percent $(42 \%)$. This is due to both values having strong relationship in the positive direction but difference between the predicted and observed data is very large, results the unusable percent being highest since if difference between forecasted and observed is more than $2^{\circ} \mathrm{C}$, it is taken as unusable.

The variation between forecasted and observed values can be reduced through local value addition to the forecasted temperature. The predicted values are higher for the monsoon and the winter season while they are lower for post monsoon and summer. The overall forecast for maximum temperature is good with good correlation coefficient for the year with the best for the post monsoon season. 
Table.1 The following $2 * 2$ contingency table is used for calculation of the various skill scores and verification of the rainfall forecast (Murphy and Wrinkler, 1987; Murphy et al., 1989)

\begin{tabular}{|l|l|l|l|}
\hline \multirow{2}{*}{ Event forecasted } & \multicolumn{3}{|l|}{ Event observed } \\
\cline { 2 - 4 } & Yes & No & Marginal total \\
\hline Yes & $\mathrm{H}(\mathrm{YY})$ & $\mathrm{F}(\mathrm{NY})$ & $\mathrm{YY}+\mathrm{NY}(\mathrm{H}+\mathrm{F})$ \\
\hline No & $\mathrm{M}(\mathrm{YN})$ & $\mathrm{Z}(\mathrm{NN})$ & $\mathrm{YN}+\mathrm{NN}(\mathrm{M}+\mathrm{Z})$ \\
\hline Marginal total & $\mathrm{YY}+\mathrm{YN}(\mathrm{H}+\mathrm{M})$ & $\mathrm{NY}+\mathrm{NN}(\mathrm{F}+\mathrm{Z})$ & $\mathrm{N}(\mathrm{YY}+\mathrm{NY}+\mathrm{YN}+\mathrm{NN}) \mathrm{N}(\mathrm{H}+\mathrm{F}+\mathrm{M}+\mathrm{Z})$ \\
\hline
\end{tabular}

Table.2 Comparative table for rainfall forecast during the year 2016-17

\begin{tabular}{|l|l|l|l|l|l|}
\hline Parameters & Monsoon & Post Monsoon & Winter & Summer & Average \\
\hline Correct (\%) & 45 & 93 & 96 & 95 & 78 \\
\hline Usable (\%) & 23 & 7 & 2 & 3 & 10 \\
\hline Unusable (\%) & 32 & 0 & 2 & 2 & 12 \\
\hline RS (\%) & 60 & 90 & 87 & 73 & 75 \\
\hline HKS & 0.238 & 0.000 & 0.482 & 0.182 & 0.247 \\
\hline HSS & 0.114 & 0.000 & 0.275 & 0.103 & 0.135 \\
\hline FAR & 0.459 & 1.000 & 0.769 & 0.875 & 0.850 \\
\hline POD & 0.930 & 0.000 & 0.600 & 0.429 & 0.537 \\
\hline Corr. Coeff. & 0.254 & 0.000 & 0.517 & -0.050 & 0.197 \\
\hline RMSE (mm) & 21.019 & 2.667 & 3.370 & 4.279 & 8.584 \\
\hline
\end{tabular}

Table.3 Comparative table for cloud cover during the year 2016-17

\begin{tabular}{|l|l|l|l|l|l|}
\hline Parameters & Monsoon & Post Monsoon & Winter & Summer & Average \\
\hline Correct (\%) & 48 & 72 & 48 & 46 & 52 \\
\hline Usable (\%) & 23 & 8 & 37 & 23 & 24 \\
\hline Unusable (\%) & 29 & 20 & 15 & 31 & 24 \\
\hline Corr. Coeff. & 0.31 & 0.49 & 0.21 & 0.28 & 0.353 \\
\hline RMSE (Octa) & 2.35 & 1.97 & 3.24 & 2.43 & 2.736 \\
\hline
\end{tabular}

Table.4 Comparative table for wind speed during the year 2016-17

\begin{tabular}{|l|l|l|l|l|l|}
\hline Parameters & Monsoon & Post monsoon & Winter & Summer & Average \\
\hline Correct (\%) & 14 & 7 & 7 & 22 & 13 \\
\hline Usable (\%) & 34 & 31 & 22 & 37 & 31 \\
\hline Unusable (\%) & 52 & 62 & 71 & 41 & 56 \\
\hline Corr. Coeff. & 0.140 & -0.020 & 0.040 & 0.330 & 0.134 \\
\hline RMSE (km/h) & 4.400 & 4.670 & 5.290 & 3.340 & 4.849 \\
\hline
\end{tabular}


Table.5 Comparative table for wind direction during the entire year 2016-17

\begin{tabular}{|l|l|l|l|l|l|}
\hline Parameters & Monsoon & Post monsoon & Winter & Summer & Average \\
\hline Correct (\%) & 48 & 43 & 28 & 42 & 41 \\
\hline Usable (\%) & 12 & 11 & 12 & 9 & 11 \\
\hline Unusable (\%) & 40 & 46 & 60 & 49 & 48 \\
\hline Corr. Coeff. & 0.34 & 0.23 & 0.07 & 0.47 & 0.304 \\
\hline RMSE (degree) & 94.49 & 132.72 & 124.54 & 97.79 & 123 \\
\hline
\end{tabular}

Table.6 Comparative table for maximum temperature during the year 2016-17

\begin{tabular}{|l|l|l|l|l|l|}
\hline Parameters & Monsoon & Post monsoon & Winter & Summer & Average \\
& & & & & \\
\hline Correct (\%) & 55 & 57 & 32 & 38 & 45 \\
\hline Usable (\%) & 15 & 28 & 27 & 20 & 22 \\
\hline Unusable (\%) & 30 & 15 & 41 & 42 & 33 \\
\hline Corr. Coeff. & 0.393 & 0.843 & 0.546 & 0.860 & 0.724 \\
\hline RMSE $\left({ }^{0} \mathrm{C}\right)$ & 2.114 & 1.356 & 2.748 & 2.436 & 2.371 \\
\hline
\end{tabular}

Table.7 Comparative table for minimum temperature during the year 2016-17

\begin{tabular}{|l|l|l|l|l|l|}
\hline Parameters & Monsoon & Post monsoon & Winter & Summer & Average \\
& & & & & \\
\hline Correct (\%) & 52 & 44 & 40 & 34 & 43 \\
\hline Usable (\%) & 22 & 30 & 23 & 17 & 22 \\
\hline Unusable (\%) & 26 & 26 & 37 & 49 & 35 \\
\hline Corr. Coeff. & 0.185 & 0.943 & 0.565 & 0.890 & 0.707 \\
\hline RMSE $\left({ }^{\circ} \mathrm{C}\right)$ & 1.715 & 1.910 & 2.165 & 2.930 & 2.389 \\
\hline
\end{tabular}

Table.8 Comparison of chi square values for maximum and minimum temperature (2016-17)

\begin{tabular}{|l|l|l|l|l|l|l|}
\hline Parameters & \multicolumn{4}{|l|}{$\left.\operatorname{Tmax}^{\mathbf{0}} \mathbf{C}\right)$} & \multicolumn{2}{l|}{$\operatorname{Tmin}\left({ }^{\mathbf{0}} \mathbf{C}\right)$} \\
\hline Seasons & $+\mathrm{ve}$ & $-\mathrm{ve}$ & Overall & $+\mathrm{ve}$ & $-\mathrm{ve}$ & Overall \\
\hline Winter & 15.87 & 15.63 & 31.50 & 7.17 & 121.45 & 128.62 \\
\hline Summer & 5.95 & 11.02 & 16.97 & 9.22 & 49.86 & 59.08 \\
\hline Monsoon & 8.27 & 8.49 & 16.77 & 4.12 & 15.58 & 19.69 \\
\hline Post monsoon & 1.34 & 3.10 & 4.43 & 3.61 & 15.77 & 19.38 \\
\hline
\end{tabular}


Fig.1 A simple flow chart for assimilation of weather data and forecasted data for dissemination to $\mathrm{AMFU}$ is shown in figure

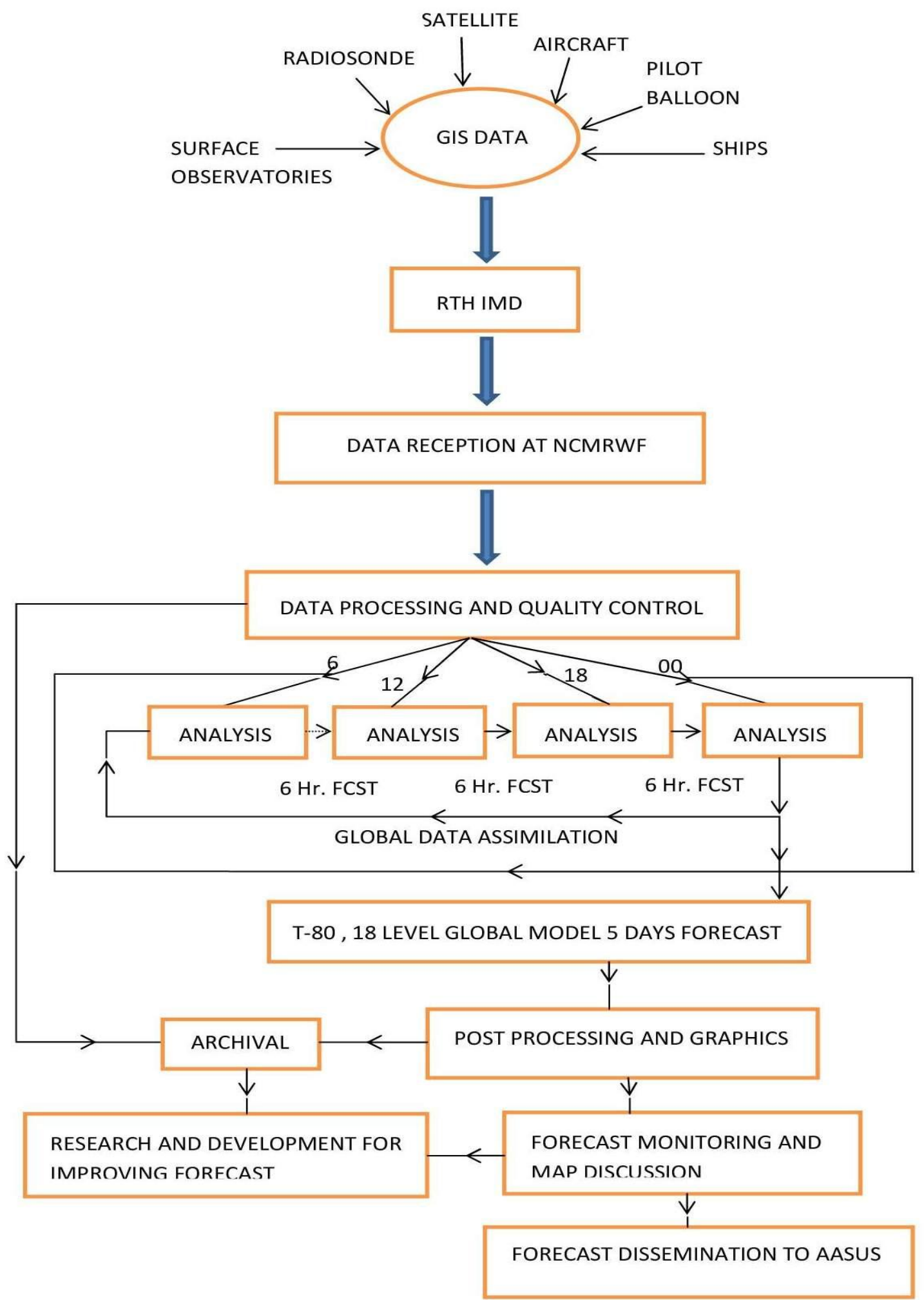


Fig.2 Variation in skill scores across the season

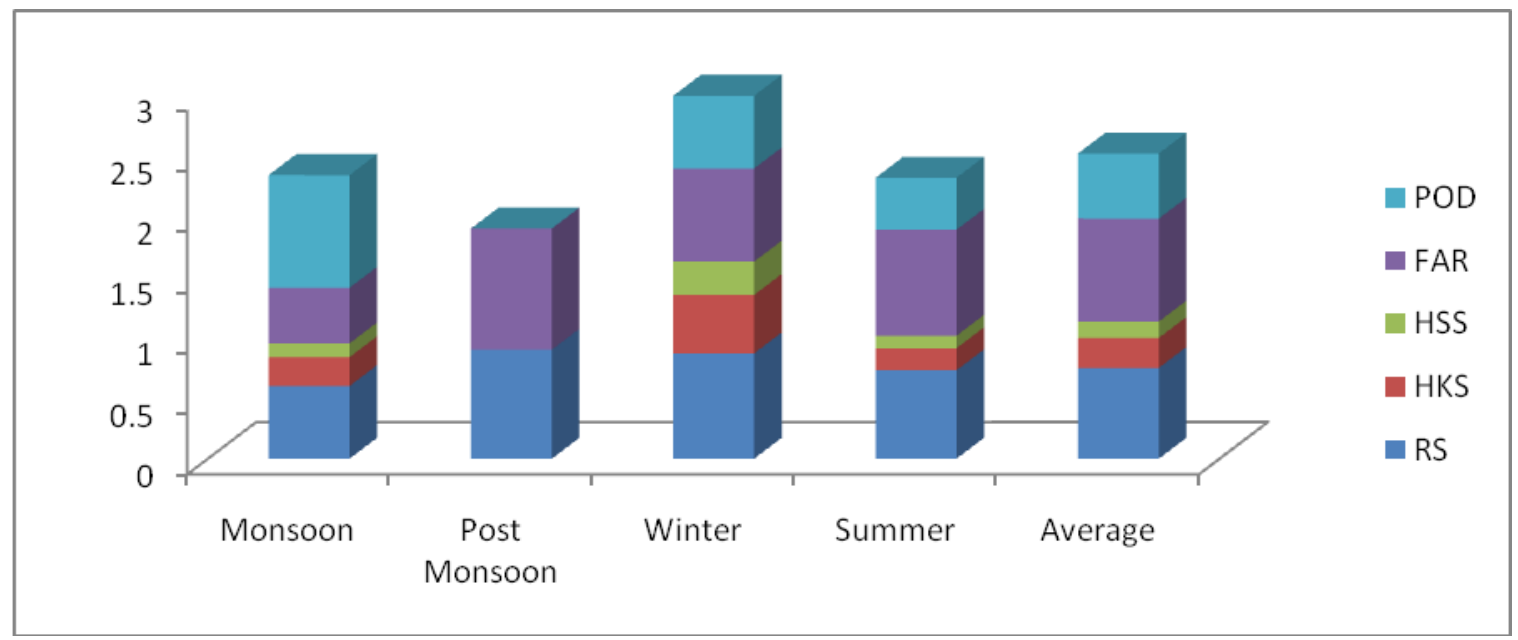

Fig.3 Wind speed usable percent variation during the year 2016-17

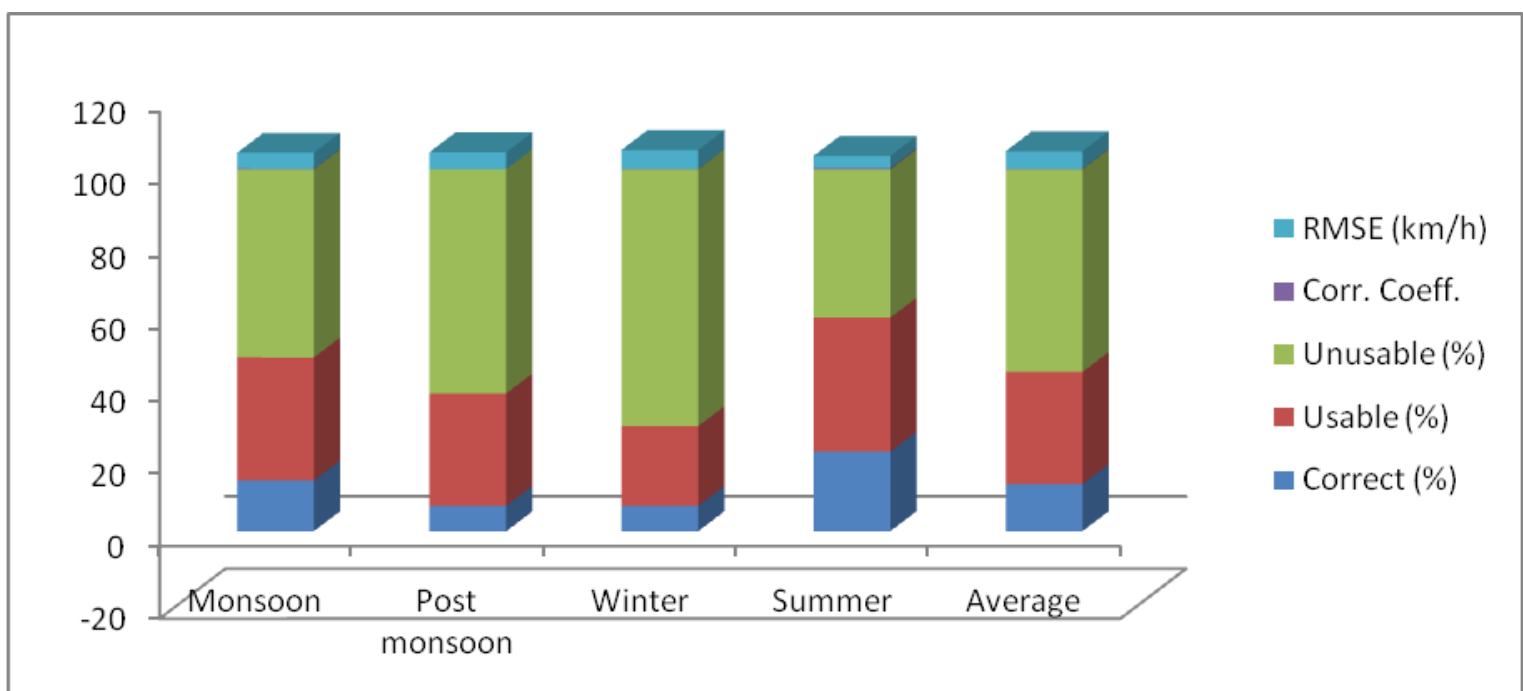

\section{Minimum temperature}

Table 7 shows the comparative and average values of different parameters during the year 2016-17. The average correct percentage of the forecast is higher, 43 followed by the average unusable percent, 35 and average usable percent, 22 percent. This represents a good forecast for the minimum temperature over the year. The average correlation coefficient with the value of 0.707 represents a strong positive correlation of the observed and predicted values. The average RMSE,
2.389 determines the low amount of error, and low difference between the data. The observed values were always higher for all the seasons as depicted. The maximum and minimum temperature gives almost similar results for the entire year. The correlation coefficient for summer season is positive as the value for it is 0.890 and this value indicates strong relation between the observed and forecasted values. This value is highest giving an extremely strong relationship between the forecasted and observed data and at the same time it has 
high unusable percent (49\%). This is due to both predicted and observed data having strong relationship in the positive direction but difference between the predicted and observed data is very large.

This result in the unusable percent being highest, since difference between forecasted and observed if more than $2^{0} \mathrm{C}$, it is taken as unusable. The variation between forecasted and observed values can be reduced through local value addition to the forecasted minimum temperature. The forecast is found to be very good for the entire year with the best for the monsoon season. Almost similar results were obtained by Gill and Babuta (2013).

\section{Comparison of chi square values for maximum and minimum temperature}

Table 8 shows the comparative and overall values of chi square for maximum and minimum temperature during the year. The chi square values clearly depict their significance by their higher total value for the winter season where large variation is seen in the predicted and observed values for both maximum and minimum temperature.

This is followed by summer season which shows a little significant difference followed by the monsoon and post monsoon with the latter having lowest significant overall chi square. Highest overall chi square during the winter season for the minimum temperature clearly depicts the large variation in the observed and predicted data values.

The rainfall forecast was good for the winter and post monsoon season on viewing the entire skill scores for the year. The correct percent depicts a good forecast but with high RMSE. The RS as always gives good representation along with HKS. However, HSS gave similar results as HKS while POD and FAR were found to be reliable scores for accuracy. If both used together are good for better accuracy detection. The cloud forecast for the entire year is good with an extremely good forecast for the post monsoon season. The forecast is good with high correct percent, moderately high correlation and low RMSE.

The wind speed and wind direction forecast for the year was very poor with high unusability, low correlation and high RMSE. Over the year the forecast for maximum and minimum temperature is good with high usability, high correlation and low RMSE.

The maximum and minimum temperature showed similar results in summer season with high unusability and high correlation which is due to the error structure introduction by IMD which needs improvement. Further, value addition of the data can also be a measure undertaken for improvement. Besides correlation coefficient and RMSE, chi square values have also provided reliable results which can also be used for better accuracy detection of temperature.

The forecast provided by IMD is good but further precision is needed because as the observations are being made the accuracy level for the most important parameter that is rainfall, wind speed and direction is poor though they are very important for various farming operations to be taken up by the farmers while for temperature too if observed not a very good forecast is provided. So, keeping in mind the following conditions further improvement in forecast is required for more effective results.

\section{Acknowledgement}

A fistful thanks to Dr. R. K. Singh and my department which provided me with the required help and information. 


\section{References}

Damrath, U., Doms, G., Friihwald, D., Heise, E., Richter, B. and Steppeler, J. 2001. Operational quantitative precipitation forecasting at the German Weather Service. Journal of Hydrology.239: 260-285.

Gadgil, S. 1989. Monsoon variability and its relationship with agricultural strategies. Paper presented at International symposium on climatic variability and food security in developing countries, pp. 249-267.

Gill, K.K. and Babuta, R. 2016. Reliability analysis of Medium Range Weather Forecasts in Central Plain Region of Punjab. Indian Journals. 56: 240-360.

Hansen, J.W. 2002. Realizing the potential benefits of climate prediction to agriculture: issues, approaches and challenges. Agricultural Systems.74: 309-330.

Khichar, M.L. and Bishnoi, O.P. 2003. Accuracy of weather forecast for western agroclimatic zone of Haryana during Kharif season. Haryana Agricultural University Journal of Research. 33(2): 97- 101.

Mummigatti U.V., Naveen N.E., Gowda P.T. and Hulihalli U.K. 2013. Validation and assessment of economic impact of agro advisories issues based on medium range weather forecast for Dharwad district of Karnataka. Agriculture Update. 8: 260-264.

Murphy, A. H., Brown, B. G. and Chen, Y. S. 1989. Diagnostic verification of temperature forecasts. American Meteorological Society. 4: 485-501.

Murphy, A.H. and Winkler, R.L. 1987. A General Framework for Forecast Verification. 115: 1330-1338.

Seeley, M.W. 1994. The future of serving agriculture with weather/climate information and forecasting: some indications and observations. Agricultural and Forest Meteorology. 69: 47-59.

\section{How to cite this article:}

Shivani Kothiyal, R.K. Singh, A.S. Nain, Himani Padalia and Gaurav Chaturvedi. 2017. Verification of Medium Range Weather Forecast for Udham Singh Nagar Region in order to Improve Methodology Followed. Int.J.Curr.Microbiol.App.Sci. 6(12): 1995-2005. doi: https://doi.org/10.20546/ijcmas.2017.612.229 